\title{
A Novel Cytotoxic C-Methylated Biflavone, Taiwanhomoflavone-B from the Twigs of Cephalotaxus wilsoniana
}

\author{
Yao-Haur Kuo, ${ }^{*, a, b}$ Shy-Yuan Hwang, ${ }^{c}$ Li-Ming Yang Kuo, ${ }^{a}$ Yi-Ling LeE, ${ }^{a, b}$ Shyh-Yuan LI, ${ }^{*, b}$ and \\ Ya-Ching SHEN ${ }^{d}$ \\ ${ }^{a}$ National Research Institute of Chinese Medicine; Shih-Pai, Taipei, 112, Taiwan, R.O.C.: ${ }^{b}$ Department of Chemistry, \\ Chinese Culture University; Taipei, 113, Taiwan, R.O.C.: ${ }^{c}$ Division of Botany, Taiwan Endemic Species Research Institute; \\ Chichi, Nantou, 552, Taiwan, R.O.C.: and ${ }^{d}$ Institute of Marine Resources, National Sun Yat-Sen University; Kaohsiung, \\ 804, Taiwan, R.O.C. Received May 20, 2002; accepted August 13, 2002
}

\begin{abstract}
A novel $C$-methylated biflavone, taiwanhomoflavone-B (1), together with known compounds, 7,4', $7^{\prime \prime}$-tri- $O$ methylamentoflavone, 6-C-methylnaringenin and apigenin-7-O- $\beta$-glucoside were isolated from an ethanolic extract of Cephalotaxus wilsoniana. The structure of 1 was elucidated on the basis of spectroscopic analysis. Taiwanhomoflavone-B is cytotoxic with $E D_{50}$ values of 3.8 and $3.5 \mu \mathrm{g} / \mathrm{ml}$, against $\mathrm{KB}$ oral epidermoid carcinoma and Hepa-3B hepatoma cells, respectively.
\end{abstract}

Key words Cephalotaxus wilsoniana; cytotoxicity; taiwanhomoflavone-B; 7,4',7"-tri-O-methylamentoflavone; 6- $C$-methylnaringenin; kamepferol; apigen-7-O- $\beta$-glucoside

Cephalotaxus wilsoniana HAYATA (Cephalotaxaceae) is an evergreen tree distributed over the middle mountains of Taiwan. Several antitumor components including hainanolide and the alkaloids homoharringtonine, harringtonine, epiwilsonine, wilsonine and cephalotaxine have been reported from Cephalotaxus spp. ${ }^{1-7)}$ Of these antitumor alkaloids, homoharringtonine has been selected as a candidate for phase II trial in the United States. Recently, we obtained a novel cytotoxic $\mathrm{C}_{31}$ biflavone, taiwanhomoflavone- $\mathrm{A}$ in addition to a known biflavone, kayaflavone, and the known compounds, harringtonolide (=hainanolide), epi-wilsonine, and diterpenes as sugiol and isopimaric acid from the EtOH extract of the stem of Cephalotaxus wilsoniana HAYATA. ${ }^{8)}$ Further investigation of the twigs of $C$. wilsoniana led to the isolation and characterization of a novel $C$-methylated biflavone, taiwanhomoflavone-B (1), along with known flavones, 7,4', $7^{\prime \prime}$-tri- $O$ methylamentoflavone (2), 6- $C$-methylnaringenin (3), and apigenin-7-O- $\beta$-glucoside (4). We report herein the structural elucidation of $\mathbf{1}$ determined by employing two dimensional (2D) NMR techniques including ${ }^{1} \mathrm{H}-{ }^{-1} \mathrm{H}$ correlation spectroscopy (COSY), ${ }^{1} \mathrm{H}-{ }^{13} \mathrm{C}$ heteronuclear multiple quantum coherence (HMQC), and ${ }^{1} \mathrm{H}-{ }^{13} \mathrm{C}$ heteronuclear multiple bond coherence (HMBC) experiments.

Taiwanhomoflavone-B (1) was obtained as a pale yellow amorphous powder. Its molecular formula $\left(\mathrm{C}_{32} \mathrm{H}_{24} \mathrm{O}_{10}\right)$ was indicated by the molecular ion $\left(\mathrm{m} / \mathrm{z} 568.1286[\mathrm{M}]^{+}\right)$in the high resolution electron impact (HR-EI)-MS spectra. The ${ }^{1} \mathrm{H}-$ and ${ }^{13} \mathrm{C}$-NMR spectra, together with IR absorption at 3400 $(\mathrm{OH}), 1660$ (conjugated $\mathrm{CO}$ ) and 1625 (aromatic) $\mathrm{cm}^{-1}$ revealed that 1 possessed two flavonoid units. The presence of signals at $\delta_{\mathrm{H}} 7.56\left(\mathrm{H}-2^{\prime}, 6^{\prime}, \mathrm{d}, J=8.5 \mathrm{~Hz}\right), \delta_{\mathrm{H}} 7.30\left(\mathrm{H}-3^{\prime}, 5^{\prime}\right.$, $\mathrm{d}, J=8.5 \mathrm{~Hz}), \delta_{\mathrm{H}} 7.98\left(\mathrm{H}-2^{\prime \prime \prime}, 6^{\prime \prime \prime}, \mathrm{d}, J=8.5 \mathrm{~Hz}\right)$ and $\delta_{\mathrm{H}} 7.27$ $\left(\mathrm{H}-3^{\prime \prime \prime}, 5^{\prime \prime \prime}, \mathrm{d}, J=8.5 \mathrm{~Hz}\right)$ in an $\mathrm{A}_{2} \mathrm{~B}_{2}$ coupling system, together with the signals of $\delta_{\mathrm{C}} 128.56\left(\mathrm{C}-2^{\prime}, 6^{\prime} ; \mathrm{C}-2^{\prime \prime \prime}, 6^{\prime \prime \prime}\right)$ and 116.93 (C-3', C-5'; C-3"', C-5'"') observed in the NMR spectra suggested two B-ring aromatic moieties in 1. In the HMBC spectrum, two aromatic signals in a singlet were assigned at H-8 and $8^{\prime \prime}$, respectively, due to the long-range coupling with C-7, 6 and C-7", 6", respectively. Then the methoxyl group at $\mathrm{C}-7^{\prime \prime}$ was sequentially confirmed. Furthermore, in compari- son with the chemical shifts of $\mathrm{C}-8\left(\delta_{\mathrm{C}} 94.99\right)$ and C-8" $\delta_{\mathrm{C}}$ 91.85), the higher chemical shift for C-8 by $c a . \delta_{\mathrm{C}} 3-4$ is consistent with that of similar flavonoids where a hydroxyl group was replaced by a methoxyl group at C-7". ${ }^{9)}$ The available evidence of C- 6 " chemical shift $\left(\delta_{\mathrm{C}} 126.56\right)$ suggests a linkage between two flavonoid units at $\mathrm{C}-4^{\prime}-\mathrm{O}-\mathrm{C}-6^{\prime \prime} .{ }^{10)}$ This corroboration also excludes the possibility of linkage of the two flavonoid units by C-4'-O-C-8". Otherwise, the chemical shift for C-6" in 1 would be changed from general $\delta_{\mathrm{C}} 98$ to 95 , due to a methoxyl group at $\mathrm{C}-7^{\prime \prime}$ position. ${ }^{9}$ )

Moreover, based on the HMBC spectrum, two carbonyl carbons at C-4 and C-4" $\left(\delta_{\mathrm{C}} 182.98,196.21\right)$ were unambiguously assigned due to long-range coupling with an olefinic $\mathrm{H}-3$ and the saturated $\mathrm{H}-2^{\prime \prime}$ and $\mathrm{H}-3^{\prime \prime}$, respectively. Together with the above evidence, 1 was deduced as a $2^{\prime \prime}, 3^{\prime \prime}$-dihydroisocrytomerin analogue except for an additional methyl signal $\left(\delta_{\mathrm{H}} 2.35\right)$ was observed in 1 . These findings revealed that 1 had a new $\mathrm{C}_{31}$ skeleton with two flavones linked by $\mathrm{C}$ $4^{\prime}-\mathrm{O}-\mathrm{C}-6$ ". After detailed examination of the HMBC spectrum of 1, the methyl group was assigned at C-6 due to the correlation between the methyl group and C-5 and C-6, and between $\mathrm{H}-8$ and C-6, respectively. Thus, the structure of 6$C$-methyl-2", 3"-dihydroisocrytomerin (1) was confirmed completely and tentatively named taiwanhomoflavone-B.

Compounds 1 and 4, hainanolide (5), epi-wilsonine (6), sugiol (7) and isopimaric acid (8) were tested against four cancer cell lines: KB, COLO-205, Hepa-3B and Hela. The bioassy data exhibited that only diterpenes $\mathbf{7}$ and $\mathbf{8}$ were inactive, whereas the other entries showed the cytotoxicity (Table 1). Not surprisingly, hainanolide (5) possessed the

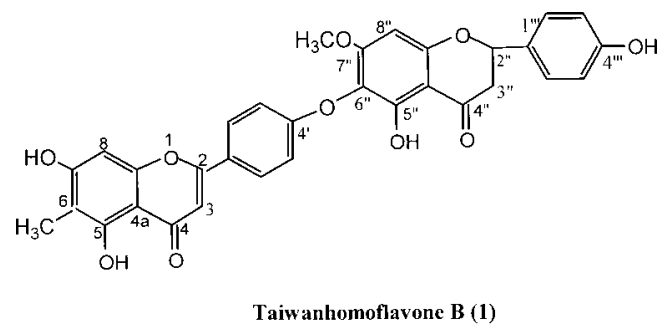


most active effect against the above four tumor cells in this experiment; it was also report to exhibit the potent cytotoxicity against several other tumor cell lines. ${ }^{11)}$ The other active

Table 1. Cytotoxicity of Compounds $\mathbf{1}, \mathbf{4}, \mathbf{5}-\mathbf{8}$ against Tumor Cell Lines

\begin{tabular}{ccccc}
\hline \hline \multirow{2}{*}{ Entries } & \multicolumn{4}{c}{ Cell line $\left(\mathrm{ED}_{50}, \mu \mathrm{g} / \mathrm{ml}\right)$} \\
\cline { 2 - 5 } & $\mathrm{KB}^{a)}$ & $\left.\mathrm{COLO}^{2} 25^{a}\right)$ & Hepa-3B $^{a)}$ & Hela $^{a)}$ \\
\hline $\mathbf{1}$ & 3.8 & $b)$ & 3.5 & $b)$ \\
$\mathbf{4}$ & 3.5 & $b)$ & 8.7 & $b)$ \\
$\mathbf{5}$ & 0.11 & $b)$ & 0.05 & 0.37 \\
$\mathbf{6}$ & 1.94 & $b)$ & $b)$ & $b)$ \\
$\mathbf{7}$ & $-c)$ & - & - & - \\
$\mathbf{8}$ & - & - & - & - \\
\hline
\end{tabular}

a) Hepa-3B: hepatoma, KB: oral epidermoid carcinoma, COLO-205: colon carcinoma, Hela: cervix carcinoma. b) No test. c) Inactive, $\mathrm{ED}_{50}>10 \mu \mathrm{g} / \mathrm{ml}$.

Table 2. ${ }^{1} \mathrm{H}$ - and ${ }^{13} \mathrm{C}-\mathrm{NMR}$ Data ${ }^{a}$ (Pyridine- $d_{5}$ ) for Compound $\mathbf{1}$

\begin{tabular}{|c|c|c|c|}
\hline Carbon & ${ }^{13} \mathrm{C}(\mathrm{ppm})$ & ${ }^{1} \mathrm{H}(\mathrm{ppm})$ & ${ }^{13} \mathrm{C}-{ }^{1} \mathrm{H}$ connectivities ${ }^{b)}$ \\
\hline 2 & $165.08 \mathrm{~s}$ & - & $\mathrm{H}-3$ \\
\hline 3 & $103.79 \mathrm{~d}$ & $6.98(\mathrm{~s})$ & - \\
\hline 4 & $182.98 \mathrm{~s}$ & - & $\mathrm{H}-3$ \\
\hline $4 a$ & $102.46 \mathrm{~s}$ & - & $\mathrm{H}-8,5-\mathrm{OH}$ \\
\hline 5 & $162.18 \mathrm{~s}$ & - & $6-\mathrm{Me}, 5-\mathrm{OH}$ \\
\hline 6 & $104.99 \mathrm{~s}$ & - & $\mathrm{H}-8,6-\mathrm{Me}, 5-\mathrm{OH}$ \\
\hline 7 & $166.21 \mathrm{~s}$ & - & $\mathrm{H}-8,6-\mathrm{Me}$ \\
\hline 8 & $94.99 \mathrm{~d}$ & $6.41(\mathrm{~s})$ & 7-OH \\
\hline $8 \mathrm{a}$ & $161.32 \mathrm{~s}$ & - & H-8 \\
\hline $1^{\prime}$ & $121.93 \mathrm{~s}$ & - & H-3, H-3', H-5' \\
\hline $2^{\prime}$ & $128.56 \mathrm{~d}$ & $7.56(\mathrm{~d}, 8.5)$ & H-6 \\
\hline $3^{\prime}$ & $116.93 \mathrm{~d}$ & $7.30(\mathrm{~d}, 8.5)$ & $\mathrm{H}-5^{\prime}$ \\
\hline $4^{\prime}$ & $162.97 \mathrm{~s}$ & - & $\mathrm{H}-3^{\prime}, \mathrm{H}-5^{\prime}$ \\
\hline $5^{\prime}$ & $116.93 \mathrm{~d}$ & $7.30(\mathrm{~d}, 8.5)$ & $\mathrm{H}-3^{\prime}$ \\
\hline $6^{\prime}$ & $128.56 \mathrm{~d}$ & $7.56(\mathrm{~d}, 8.5)$ & $\mathrm{H}-2^{\prime}$ \\
\hline $2^{\prime \prime}$ & $79.23 \mathrm{~d}$ & $5.44(\mathrm{dd}, 13,3)$ & $\mathrm{H}-3^{\prime \prime}$ \\
\hline $3^{\prime \prime}$ & $43.35 \mathrm{t}$ & $\begin{array}{l}2.80(\mathrm{dd}, 17.0,3) \\
3.20(\mathrm{dd}, 17.0,13.0)\end{array}$ & $\mathrm{H}-2^{\prime \prime}$ \\
\hline $4^{\prime \prime}$ & $196.21 \mathrm{~s}$ & - & $\mathrm{H}-3^{\prime \prime}$ \\
\hline $4^{\prime \prime} \mathrm{a}$ & $106.27 \mathrm{~s}$ & - & $\mathrm{H}-8^{\prime \prime}$ \\
\hline $5^{\prime \prime}$ & $153.92 \mathrm{~s}$ & - & - \\
\hline $6^{\prime \prime}$ & $126.56 \mathrm{~s}$ & - & $\mathrm{H}-8^{\prime \prime}$ \\
\hline $7^{\prime \prime}$ & $159.13 \mathrm{~s}$ & - & 7"-OMe, H- $8^{\prime \prime}$ \\
\hline $8^{\prime \prime}$ & $91.85 \mathrm{~d}$ & $6.91(\mathrm{~s})$ & - \\
\hline $8^{\prime \prime} \mathrm{a}$ & $154.65 \mathrm{~s}$ & - & $\mathrm{H}-8^{\prime \prime}$ \\
\hline $1^{\prime \prime \prime}$ & $132.90 \mathrm{~s}$ & - & H-3"', $5^{\prime \prime \prime}, 2^{\prime \prime}, 3^{\prime \prime}$ \\
\hline $2^{\prime \prime \prime}$ & $129.01 \mathrm{~d}$ & $7.98(\mathrm{~d}, 8.5)$ & H-6"', H-2" \\
\hline $3^{\prime \prime \prime}$ & $115.46 \mathrm{~d}$ & $7.27(\mathrm{~d}, 8.5)$ & $\mathrm{H}-5^{\prime \prime \prime}$ \\
\hline $4^{\prime \prime \prime}$ & $159.05 \mathrm{~s}$ & - & $\mathrm{H}-3^{\prime \prime \prime}, 5^{\prime \prime \prime}$ \\
\hline $5^{\prime \prime \prime}$ & $115.46 \mathrm{~d}$ & $7.27(\mathrm{~d}, 8.5)$ & $\mathrm{H}-3^{\prime \prime \prime}$ \\
\hline $6^{\prime \prime \prime}$ & $129.01 \mathrm{~d}$ & $7.98(\mathrm{~d}, 8.5)$ & $\mathrm{H}-2^{\prime \prime \prime}, \mathrm{H}-2^{\prime \prime}$ \\
\hline $6-\mathrm{Me}$ & $7.55 \mathrm{q}$ & $2.35(\mathrm{~s})$ & —c) $^{\prime}$ \\
\hline $7^{\prime \prime}-\mathrm{O}-\mathrm{Me}$ & $56.60 \mathrm{~s}$ & $3.80(\mathrm{~s})$ & -c) \\
\hline
\end{tabular}

a) All assignments $\left({ }^{13} \mathrm{C} ; 75.5 \mathrm{MHz}\right.$, multiplicity; $\left.{ }^{1} \mathrm{H} ; 300 \mathrm{MHz}\right)$ are based on one dimensional (1D) and two dimensional (2D) NMR experiments, including COSY 90, HETCOR, and HMBC spectra. b) ${ }^{1} \mathrm{H}-{ }^{13} \mathrm{C}$ long-range correlation (HMBC) corresponded to two or three bonds connectivities. c) These assignments were explained in the text. principles isolated from the titled plant as well as their structure-activity relationships are still under investigation.

\section{Experimental}

General Experimental Procedures ${ }^{1} \mathrm{H}$ - and ${ }^{13} \mathrm{C}-\mathrm{NMR}$ spectra were recorded at 300.13 and $75.46 \mathrm{MHz}$, respectively, on a Bruker $300 \mathrm{AC}$ spectrometer. The spectra of heteronuclear correlation, HMBC was established by the coupling of $8 \mathrm{~Hz}$. Electron impact (EI)-MS and FAB-MS were performed on a JEOL SX-102A instrument. Si gel (Merck 70-230 mesh) was used for column chromatography, and precoated Si gel (Merck 60F-254) plates were used for TLC. HPLC was accomplished on an SPD-6AV liquid chromatograph using a preparative $\mathrm{C}_{18}$ column. Melting points were determined on a Fisher-Johns apparatus and are uncorrected.

Plant Material Twigs of Cephalotaxus wilsoniana were collected in July 1998 in Taipei, Taiwan, R.O.C. A voucher specimen is deposited at the National Research Institute of Chinese Medicine, Shih-Pai, Taipei.

Extraction and Isolation The dried twigs of $C$. wilsoniana $(5.7 \mathrm{~kg})$ were extracted exhaustively with ethanol. An EtOH extract ( $160 \mathrm{~g})$ was extracted successively with $n$-hexane and $\mathrm{CHCl}_{3}$. The $\mathrm{CHCl}_{3}$ extract was chromatographed by column chromatography over $\mathrm{Si}$ gel and eluted with $n$ hexane-EtOAc and EtOAc to give 7 fractions. The bioactive fr. 4 (nhexane $: E t O A c=3: 1)$ was further separated by HPLC $\left(5 \mathrm{C}_{18}, 250 \times 10 \mathrm{~mm}\right)$ with $\mathrm{MeOH}-\mathrm{H}_{2} \mathrm{O}(8.5: 1)$ to furnish taiwanhomoflavone-B (1) $(9 \mathrm{mg})$.

Taiwanhomoflavone-B (1): Pale yellow powder; IR $v_{\max }(\mathrm{KBr}) 3400$ $(\mathrm{OH}), 1660$ (conjugated $\mathrm{CO}$ ) and 1625 (aromatic) $\mathrm{cm}^{-1} ;{ }^{1} \mathrm{H}$ - and ${ }^{13} \mathrm{C}-\mathrm{NMR}$, see Table 2; FAB-MS $m / z 567$ [M-H] $^{+}$; HR-EI-MS $m / z 568.1286[\mathrm{M}]^{+}$ (Calcd for $\mathrm{C}_{32} \mathrm{H}_{24} \mathrm{O}_{10}, 568.1369$ ).

Cytotoxicity Assay An in vitro cytotoxicity assay was performed as previously described. ${ }^{12}$

Acknowledgments The authors thank the National Science Council, R.O.C. (NSC 88-2314-B-077-007) for financial support to Y. H. Kuo. We also thank Mr. Shih-Jen Wang, NSC Regional Instrument Center of HSIN$\mathrm{CHU}$, for measuring the FABMS data.

\section{References and Notes}

1) Powell R. G., Weisleder D., Smith C. R., Jr., Wolff I. A., Tetrahedron Lett., 1969, 4081— 4084 (1969).

2) Powell R. G., Weisleder D., Smith C. R., Jr., Rohwedder W. K., Tetrahedron Lett., 1970, 815-818 (1970).

3) Khan N. U., Ilyas M., Rahman W., Okigawa M., Kawano N., Phytochemistry, 10, 2541—2545 (1971).

4) Powell R. G., Mikolajczak K. L., Weisleder D., Smith C. R., Jr., Phytochemistry, 11, 3317-3320 (1972).

5) Sun N., Xue Z., Liang S., Huang L., Acta Pharmaceut. Sinica, 1, 3943 (1979).

6) Qiu M., Lu B. M. X., Nie R., Acta Botanica Yunnanica, 1, 97—99 (1997).

7) Furukawa H., Itoigawa M., Haruna M., Jinno Y., Ito K., Lu S. T., Yakugaku Zasshi, 96, 1373-1377 (1976).

8) Kuo Y. H., Lin C. H., Hwang S. Y., Shen Y. C., Lee Y. L., Li S. Y., Chem. Pharm. Bull., 48, 440-441 (2000).

9) Markham K. R., Sheppard C., Geiger H., Phytochemistry, 26, 33353337 (1987).

10) Silva G. L., Chai H., Gupta M. P., Farnsworth N. R., Cordell G. A., Pezzuto J. M., Beecher C. W. W., Kinghorn A. D., Phytochemistry, 40, 129-134 (1995).

11) Sun N., Zhao Z., Chen R., Lin W., Zhou Y., Acta Pharmaceut. Sinica, 16, 233-234 (1981).

12) Kuo Y. H., Huang H. C., Kuo Yang L. M., Chen C. F., J. Org. Chem., 64, 7023-7027 (1999). 